\title{
BMJ Open Randomised, double-blind, multicentre, phase I/II dose escalation and expansion trial of GR1501 in patients with plaque psoriasis: study protocol
}

Wenliang Dong (10 , ${ }^{1,2}$ Xiaoyan Nie, ${ }^{2}$ Jiaxue Wang, ${ }^{1,2}$ Lin Xia, ${ }^{3}$ Lin Cai, ${ }^{4}$ Qian Wang, ${ }^{1}$ Wei Wang, ${ }^{5}$ Weixing Fu, ${ }^{5}$ Qi Wang, ${ }^{1,2}$ Tiantian Shen, ${ }^{3}$ Huaying Fan, ${ }^{6}$ Suping Niu, ${ }^{6}$ Yimin Cui, ${ }^{7}$ Qingshan Zheng, ${ }^{8}$ Jianzhong Zhang, ${ }^{4}$ Yi Fang ${ }^{1}$

To cite: Dong W, Nie X, Wang J, et al. Randomised, double-blind, multicentre, phase I/II dose escalation and expansion trial of GR1501 in patients with plaque psoriasis: study protocol. BMJ Open 2020;10:e039067. doi:10.1136/ bmjopen-2020-039067

- Prepublication history and additional material for this paper is available online. To view these files, please visit the journal online (http://dx.doi.org/10. 1136/bmjopen-2020-039067).

WD and XN contributed equally.

Received 06 April 2020 Revised 09 0ctober 2020 Accepted 16 October 2020
Check for updates

\section{(C) Author(s) (or their} employer(s)) 2020. Re-use permitted under CC BY-NC. No commercial re-use. See rights and permissions. Published by BMJ.

For numbered affiliations see end of article.

Correspondence to

Yi Fang; phaseistudy@163.com

\section{ABSTRACT}

Introduction Psoriasis is a life-long, immune-mediated disease that greatly reduces the quality of life of patients. Plaque psoriasis is the most common form of psoriasis. Treatment options for plaque psoriasis with good tolerance and sufficient response remain profoundly limited. Based on mechanistic findings that suggest the key pathogenic role of interleukin (IL)-17 in plaque psoriasis, we hypothesise that GR1501, a new monoclonal antibody (IL-17A targeted), will be an efficacious treatment for plaque psoriasis. This phase $\mathrm{I} / \mathrm{II}$ trial aims to evaluate the safety, tolerability, pharmacokinetics, immunogenicity and preliminary efficacy of GR1501.

Methods and analysis A multicentre, randomised, double-blind, phase $\mathrm{l} / \mathrm{Il}$ dose escalation and expansion trial will be conducted at four hospitals in China. In total, 226 patients with plaque psoriasis will be enrolled in the study, with 46 cases in the dose-escalation stage and 180 cases randomised to GR1501 or the placebo in a 3:1 ratio in the expansion cohort. The primary outcomes are safety and tolerability; the secondary outcomes include pharmacokinetics, immunogenicity and efficacy.

Ethics and dissemination The study is in accordance with the Declaration of Helsinki, and the ethics approvals of the protocol have been obtained from the ethics committees of all participating centres, including Peking University People's Hospital, Chinese PLA General Hospital, The First Affiliated Hospital, College of Medicine, Zhejiang University and the Second Xiangya Hospital of Central South University. The findings of the study will be presented in published journals or at scientific conferences or meetings.

Trial registration number ChiCTR1800017956.

\section{INTRODUCTION}

Psoriasis is a common life-long immunemediated genetic disease characterised by raised areas of abnormal skin and/or joint symptoms, with a prevalence of $2 \%-4 \% .^{12}$ Plaque psoriasis is the most common type of psoriasis, accounting for approximately $90 \%$ of all psoriasis cases. ${ }^{1}$ Patients with psoriasis report a tremendous economic and

\section{Strengths and limitations of this study}

- In this study, we will use the combination design of phase I/II dose escalation and expansion, an innovative study design, which is intended to accelerate the development of new drugs from bench to bedside by seamlessly proceeding from the initial determination of a potentially effective dose in subject cohorts using the phase IIb trial approach.

- Choosing no observed adverse effect level as the method of determining the starting dose may lead to the inability to account for the effect of pharmacological pathways on dose selection.

- The inclusion of seriously ill patients or those with concomitant diseases and medications as study subjects may bring potential risks.

psychosocial burden, with tens of thousands of dollars being spent annually on the disease and a significant reduction in physical activity, cognitive function and quality of life. ${ }^{34}$ Psoriasis results from a T-cellmediated immune inflammatory response, which is mainly driven by the TNF- $\alpha$ pathway and interleukin-23 (IL-23)/Th17/IL-17 axis pathway. In the IL-23/Th17/IL-17 axis pathway, progression of psoriasis leads to an increase in the level of IL-23, which then drives the differentiation of Th17 cells. Activated Th17 cells produce several mediators such as IL-17A, which induces keratinocyte proliferation and other hallmark features of psoriasis. ${ }^{5-8}$ IL-17A is a proinflammatory cytokine mainly produced by activated $\mathrm{T}$ cells. Many studies have shown that IL-17A plays an important role in the pathogenesis of various inflammatory diseases: plaque psoriasis, ankylosing spondylitis and rheumatoid arthritis. ${ }^{9} 10$

Currently, the available treatments for patients with psoriasis include phototherapy, traditional systemic therapy and targeted 
biologics. ${ }^{11}$ The management of plaque psoriasis has been revolutionised in the past decades with the advent of biologic agents owing to their high efficacy and tolerability. ${ }^{12}$ Anti-IL-17 agents are one of the most efficacious biologic agents available for psoriasis. Currently, there are three anti-IL-17 agents on the market: secukinumab, ixekizumab and brodalumab.

GR1501, a fully human IgG4 monoclonal antibody, is a new product targeted at IL-17A with high affinity and selectivity for IL-17A; it is to be administered subcutaneously. The objective of this study is to evaluate the safety, tolerability, pharmacokinetics (PK), immunogenicity and preliminary efficacy of GR1501 via a phase I/II dose escalation and expansion clinical trial of GR1501 in patients with moderate to severe plaque psoriasis. The study will provide baseline data for the next phase of clinical trials.

The study protocol consists of two stages: the doseescalation stage and the expansion cohort stage. In the dose-escalation stage, the primary outcome is the safety and tolerability of GR1501. The secondary outcome includes the PK characteristics, immunogenicity and efficacy of GR1501 after single or multiple subcutaneous injections. The expansion cohort will help obtain more reliable outcomes for safety and tolerability as well as toxicity estimates and efficacy outcomes in a larger population.

\section{METHODS AND ANALYSIS \\ Study design}

This is a randomised, double-blind, multicentre, phase I/ II dose escalation and expansion trial in China, aiming to evaluate the safety, tolerability, immunogenicity, PK and efficacy of GR1501 in patients with moderate to severe plaque psoriasis. In the phase I/II trial, 46 patients will be enrolled in the dose escalation cohorts who will receive dose from 10 to $200 \mathrm{mg}$ with single-ascending dosing (SAD) and multiple ascending dosing (MAD). The study will also have 180 patients enrolled in the expansion cohort, who will receive the following doses: 100, 150 and $200 \mathrm{mg}$.

\section{Preclinical data (unpublished data)}

Preclinical studies have demonstrated that there is a linear correlation between the drug concentration and time after a single dose of 3,10 or $30 \mathrm{mg} / \mathrm{kg}$ of GR 1501 in cynomolgus monkeys. There was no statistically significant difference in the time to reach the peak concentration in serum $\left(\mathrm{T}_{\max }\right)$ and half-life $\left(\mathrm{t}_{1 / 2}\right)$ for multiple doses $(10 \mathrm{mg} / \mathrm{kg}$, once a week, four times in a row) when compared with the same dose on single administration. However, the peak concentration in serum $\left(\mathrm{C}_{\max }\right)$ and the drug exposure level were higher with multiple doses. The accumulation index after continuous administration was $4.03 \pm 1.46$. The absolute bioavailability was 0.77 . GR1501 was mainly excreted through the urine. There were mainly drug prototypes in the serum and small molecular metabolites in the urine. Drugs and their metabolites could not easily cross the blood-brain barrier.

A preclinical toxicity trial showed that in the range of $35-400 \mathrm{mg} / \mathrm{kg}$ dose, there was no obvious toxicity after a single subcutaneous injection of GR1501 in cynomolgus monkeys, and the maximum tolerated dose (MTD) was $\geq 400 \mathrm{mg} / \mathrm{kg}$. There was no obvious related toxic reaction and local irritation after an escalating dose of 15, 50 and $150 \mathrm{mg} / \mathrm{kg}$ for 13 weeks (once a week for a total of 14 times), followed by drug withdrawal for 6 weeks in cynomolgus monkeys. The no observed adverse effect level (NOAEL) was $150 \mathrm{mg} / \mathrm{kg}$. In other preclinical studies, haemolysis and coagulation, local irritation and positive antidrug antibody cases were not observed in cynomolgus monkeys.

\section{Dosing rationale}

The starting dose is based on three aspects. First, referring to the NOAELs in the tested animal species, converting NOAELs to human equivalent dose, and selecting the most appropriate animal species, the starting dose should be $364 \mathrm{mg}$ based on safety considerations. Second, referring to the $1 / 10$ clinical dose of drugs with the same pharmacological target, secukinumab and ixekizumab, the starting dose should be $15 \mathrm{mg}$ or $30 \mathrm{mg}$ and $8 \mathrm{mg}$ or $16 \mathrm{mg}$, respectively. Third, the starting doses of secukinumab and ixekizumab in the dose-escalation trial were $0.3 \mathrm{mg} \cdot \mathrm{kg}^{-1}$ and $5 \mathrm{mg}$, respectively. In summary, the starting dose of GR1501 will be $10 \mathrm{mg}$ based on the formulation specifications and our preclinical data.

The MTD for humans is based on the $1 / 5-1 / 2$ principle of the MTD for long-toxicity tests in animals. The MTD can be considered to be greater than $1800 \mathrm{mg}$, based on a healthy per capita weight of $60 \mathrm{~kg}$. Considering the clinical doses of secukinumab and ixekizumab and according to the preclinical study results, the product is expected to be well tolerated; thus, the MTD is tentatively set at $200 \mathrm{mg}$. In summary, according to a modified Fibonacci sequence and the determined starting dose and maximum tolerable dose, the doses in the dose-escalation stage will be 10, 30, 60, 100, 150 and $200 \mathrm{mg}$.

\section{Dose escalation}

The patients will first receive a single dose and will be observed for 6 weeks (SAD). Then, the patients in the $60-150 \mathrm{mg}$ dose group will continue to receive 12 weeks of consecutive multiple doses (MAD) once every 2 weeks. They will also be observed for 10 weeks after the 12-week administration. A single dose of the next dose group will be started on the premise that a single dose of the previous dose group is safely tolerated (single dose observation for at least 2 weeks) in half of the patients. Multiple doses of the previous dose group for 12 consecutive weeks will be started on the premise that a single dose of the next dose group is safely tolerated (single dose observation for at least 2 weeks) in half of the patients. Refer to figure 1 for details. 


\begin{tabular}{lllllll}
\hline Group & 1 & 2 & 3 & 4 & 5 & 6 \\
\hline Increasing proportion & Starting dose & 200 & 100 & 66.7 & 50 & 33.3 \\
Administration dose & 10 & 30 & 60 & 100 & 150 & 200 \\
Administr ation cycle & Single dose & Single dose & $\begin{array}{l}\text { Single } \\
\text { dose+Q2W }\end{array}$ & $\begin{array}{l}\text { Single } \\
\text { dose+Q2W }\end{array}$ & $\begin{array}{l}\text { Single } \\
\text { dose+Q2W }\end{array}$ & Single dose \\
$\begin{array}{l}\text { Number of cases in the } \\
\text { trial group }\end{array}$ & 2 & 2 & 9 & 9 & 9 & 9 \\
\hline $\begin{array}{l}\text { Number of cases in the } \\
\text { placebo group }\end{array}$ & 1 & 1 & 1 & 1 & 1 & 1 \\
\hline
\end{tabular}

Figure 1 Schematic overview of the dose-escalation stage.

\section{Expansion cohort}

A randomised, double-blind, placebo-parallel, multicentre study in the expansion cohort will be conducted after the dose-escalation study. One hundred and eighty patients will be randomly divided into the $100 \mathrm{mg}$ arm, $150 \mathrm{mg}$ arm, $200 \mathrm{mg}$ arm and placebo arm in a ratio of 1:1:1:1 and administrated the drug for 12 consecutive weeks (multiple-dose period). At the 12th week, the patients in the active arm with effective outcomes (effective: relative baseline Psoriasis Area and Severity Index (PASI) score reduction $\geq 75 \%$; partially effective: relative baseline PASI score reduction $\geq 50 \%$ but $<75 \%$; ineffective: relative baseline PASI score reduction $<50 \%$ ) will be randomly assigned to the $200 \mathrm{mg}$ Q4W arm and $200 \mathrm{mg}$ Q12W arm in a ratio of 2:1; they will thus enter the longterm dose administration period for 40 weeks. At week 12 , the patients in the placebo arm and those in the active arm who have partially effective and ineffective outcomes will receive $200 \mathrm{mg}$ Q4W for 40 consecutive weeks. There will be a 4-week follow-up after the end of the long-term dose administration period (follow-up period) after a total of 52 weeks of administration. Refer to figure 2 for details.

\section{Criteria for the discontinuation of study treatment}

In the dose-escalation stage, the investigator will stop the study if one or more patients in the test group experience grade 3 or higher adverse events related to the study drug, if half or more patients in the test group experience grade 2 adverse events related to the study drug, or when the maximum dose is reached. In addition, the sponsor, the administrative department or the ethics committee may discontinue the study if necessary. In the expansion cohort, if one or more patients in the $200 \mathrm{mg}$ group experience grade 3 or higher adverse events related to the study drug at any time point or if half or more patients in the $200 \mathrm{mg}$ group experience grade 2 adverse events associated with the study drug at any time point, the data safety supervisory committee will hold a meeting to review case information, assess risks and make suitable recommendations (eg, termination, suspension and continuation).

\section{Study population}

Adults (18-65 years old) with a diagnosis of chronic stable plaque psoriasis will be enrolled. Patients with other types of psoriasis or patients who have recently received relevant treatments or drugs will be excluded from the study. In the course of the study, patients will be withdrawn from the study if at least one of the following occurs: poor compliance to medicines, other coadministered drugs or

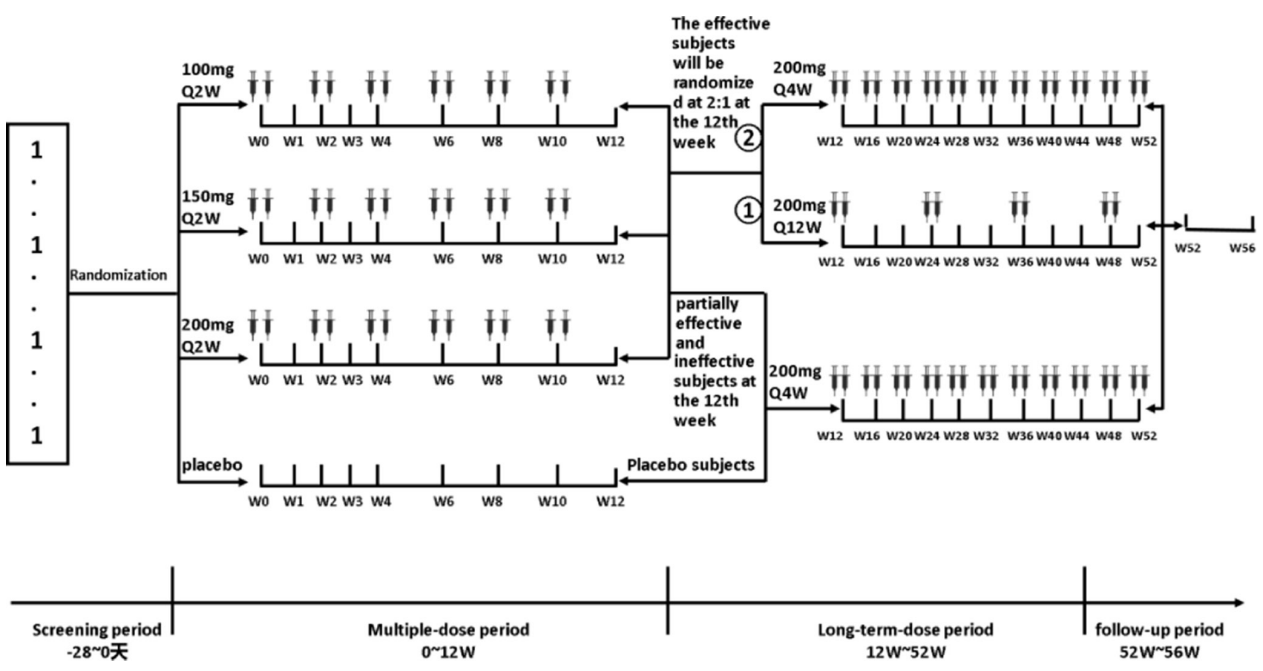

Figure 2 Schematic overview of the expansion cohort. 
foods affect the study drug tolerance judgement and drug metabolism process, voluntary withdrawal from the study, occurrence of serious adverse events whose causality cannot be determined by the investigator, the investigator determines that the treatment is invalid or it is otherwise inappropriate to continue the trial. The inclusion and exclusion criteria are detailed in the online supplemental appendix 1.

\section{Randomisation and blinding}

Randomisation and blinding will be performed by an independent investigator who will not be involved in the data management and statistical analysis of the trial. The DAS electronic interactive web response system (DAS for IWRS) will be used to generate randomisation codes and allocate treatments. Randomisation will be performed using the SAS (V.9.4) software. The expansion cohort will be established using a dynamic randomised method, considering body weight ( $\geq 75$ or $<75 \mathrm{~kg}$ ), use of biological agents for plaque psoriasis (yes or no) and static physicians global assessment (sPGA) score $(3 \leq$ sPGA $<4$ or sPGA $\geq 4$ ).

The patients will be allocated a numbered treatment pack, which will contain all the drugs or placebos needed to complete a course of the trial treatment. All trial drugs will be packaged identically and identified only by numbers. Patients, investigators and study site personnel will remain blinded to the randomisation codes until the end of the trial.

Accidental unblinding of the study participants will be informed and explained to the clinical trial unit leader, clinical research associate and trial statistician within 24 hours. All identified unblinded cases before the end of the trial will be considered drop-out cases, and such data will be kept intact.

\section{Combination therapy}

In the course of the study, the use of systemic and topical treatments for psoriasis will not be permitted. These include methotrexate, cyclosporine, etretinate, hormones and phototherapy.

The drugs permitted to be used by the study participants in the course of the study include hypolipidemic drugs, antihypertensive drugs, antidepressants, oral type II insulin drugs, oestrogen replacement therapy, antihistamines for pruritus, antacids, non-steroidal anti-inflammatory drugs, histamine $\mathrm{H}_{2}$ receptor antagonists, proton pump inhibitors, calcium supplements and topical ophthalmic drugs. Moreover, the study participants will be permitted to use topical antibiotics (for secondary infections), tar-containing shampoos, paracetamol or aspirin, topical moisturisers/emollients (not containing urea, hormones), bath oils and salicylates. The participants will be required to consult the investigator when using any other drugs or dietary supplements.

\section{Evaluation criteria for outcome}

The safety and tolerability evaluation criteria will include adverse events (the severity of the adverse events will be graded according to the Common Terminology Criteria for Adverse Events V.4.0. online supplemental appendix 2), laboratory tests, vital signs and physical examinations. The PK evaluation criteria will include $\mathrm{C}_{\max }, \mathrm{AUC}_{0-\mathrm{t}}, \mathrm{AUC}_{0}$ $C, \mathrm{~T}_{\max }, \mathrm{t}_{1 / 2 z}, \mathrm{~V}_{z}, \mathrm{CL}_{z}, \lambda_{z}, \mathrm{MRT}_{0-\mathrm{t}}, \mathrm{MRT}_{0-\infty}$ and $\mathrm{AUC}_{-\% \text { Extrap }}$ in $\mathrm{SAD}$ and $\mathrm{C}_{\text {max ss }}, \mathrm{AUC}_{0-\mathrm{tss}}, \mathrm{AUC}_{0-\infty, \mathrm{ss}}, \mathrm{AUC}_{\mathrm{ss}}, \mathrm{C}_{\mathrm{av}}, \mathrm{T}_{\text {max }}{ }^{-} \mathrm{t}_{1 / 2 \mathrm{z}}, \mathrm{V}_{\mathrm{z}}$, $\mathrm{CL}_{\mathrm{ss}}, \lambda_{z}, \mathrm{AUC}_{\text {\% Extrap }}, \mathrm{MRT}_{0-\infty, \mathrm{ss}}$ and $\mathrm{R}_{\mathrm{ac}}$ in MAD. The immunogenicity evaluation criteria will include the antidrug antibody positive rate before and after administration. The efficacy will be determined according to PASI 75, PASI 90, sPGA ' 0 ' or ' 1 '; the ratio of PASI 75 , PASI 90, sPGA ' 0 ' or ' 1 ' at different time points in the different administration arms will be compared (PASI 75/90: the proportion of patients whose PASI score was reduced by at least $75 \% / 90 \%$ compared with the baseline level. sPGA ' 0 ' (clear) or ' 1 ' (minimum): the proportion of patients whose sPGA is 0 or 1 and have at least a two-point improvement).

The PASI score is calculated as follows :

PASI $\quad($ head $)=0.1 \quad$ (erythema + infiltration + desquama tion) $\times$ skin lesion area;

PASI $($ upper limb) $=0.2$ (erythema+infiltration+desquamation) $\times$ skin lesion area;

PASI $\quad$ (trunk) $=0.3 \quad$ (erythema+infiltration+desquamation) $\times$ skin lesion area;

PASI $($ lower $\operatorname{limb})=0.4$ (erythema+infiltration + desquamation) $\times$ skin lesion area;

PASI total score= PASI (head) +PASI (upper limb) + PASI (lower limb) + PASI (trunk).

\section{Statistical analysis}

The full analysis set will include all patients who were randomly allocated, and had received $\geq 1$ dose of study drug with evaluation data. The safety set will include all patients who received $\geq 1$ dose of study drug with safety evaluation data. The PK concentration set will include all patients who received study drug dose and had $\geq 1$ quantifiable plasma concentration collected after dosing. The PK parameter set will include all patients who received the study drug dose and had $\geq 1$ valid PK parameter collected after dosing. Continuous variables will be described as the means, SDs quartile, minimum and maximum. Dichotomous and categorical variables will be described as the frequency and percentage. Statistical analysis will be performed using SAS V.9.4. Significance will be set at a $p$ value of 0.05 .

\section{Patient and public involvement}

The patients and the public will not be involved in the design, conduct, reporting or dissemination plans of the research. 


\section{DISCUSSION}

Psoriasis is a common inflammatory skin disease that is chronic and lifelong. Many patients remain untreated, do not respond to therapy or suffer from toxicities associated with traditional treatment, including phototherapy and systemic therapy. With the recent rapid development of biotechnology, the emergence of biological agents has led to safer and more effective treatment options for patients with psoriasis. The biological agents for the treatment of psoriasis include TNF- $\alpha$ inhibitors, IL-12/IL-23 inhibitors, IL-23 inhibitors and IL-17 inhibitors. Head-to-head studies have shown that the safety and anti-inflammatory efficacy of IL-17 inhibitors are superior to those of TNF- $\alpha$ inhibitors such as etanercept and adalimumab. ${ }^{13-15} \mathrm{~A}$ systematic review and network meta-analysis including 28 studies showed that IL-17 inhibitors had superior efficacy to IL-12/IL-23 and IL-23 inhibitors. ${ }^{16}$ Moreover, there is a negative correlation between patient body weight and response to treatment for many biologics such as TNF- $\alpha$ inhibitors and IL-12/IL-23 inhibitors, which means that overweight patients cannot achieve the same efficacy under the same dose as normal-weight patients. ${ }^{17}$ However, IL-17 inhibitors do not present such problems, and all patients could obtain similar treatment outcomes regardless of their weight. Finally, IL-17 inhibitors have lower immunogenicity than other biologics, especially TNF inhibitors, indicating that its risk of causing unanticipated pharmacological effects and adverse drug reactions would be reduced. ${ }^{18-20}$ GR1501 is a humanised IgG4 monoclonal antibody with high affinity for human IL-17 and high specificity to IL-17A $\left(\mathrm{K}_{\mathrm{D}}=1.75 \times 10^{-10} \mathrm{M}\right)$. The similarity and differences between GR1501 and all approved IL-17 inhibitors are shown in the online supplemental appendix 3. GR1501 is expected to represent a therapeutic option for patients who are candidates for initial systemic therapy as well as those who have failed to respond or are intolerant to current therapies on the market.

The purpose of this study is to evaluate the safety, tolerability PK, immunogenicity and efficacy of GR1501 to guide the design of clinical trials in the next phases. Three biological agents for the management of psoriasis, secukinumab, ${ }^{21} 22$ ixekizumab and brodalumab, ${ }^{23} 24$ are already on the market. We compared our protocol with the protocols for these three drugs (online supplemental appendix 4) and observed that our study will accelerate the drug development process by seamlessly proceeding from the initial dose to a determined dose as is done in phase IIb trials. ${ }^{25}$ Besides, considering the good safety profile of GR1501 from our preclinical data, to obtain PD/ biomarker and surrogate data, to benefit patients as early as possible and to improve external validity, patients with related diseases will be included as subjects in our study; this could improve the generalisability of the trial results and speed up the drug development process. Moreover, in the expansion cohort, long-term (52 week) safety and efficacy assessments, which are not usually performed in phase I or phase II studies, will be performed.
We acknowledge the several limitations of our study. The maximum recommended starting dose (MRSD) in this study will be determined using the NOAEL method. NOAEL can be used to determine a relatively safe MRSD, but it may not take into account the pharmacological pathway of some important macromolecular agents. Owing to the differences between humans and animal species, especially in $\mathrm{PD}$, affinity and efficacy, the prediction of MRSD for macromolecular drugs by the NOAEL method is often not accurate. ${ }^{26}$ The European Medicines Agency recommended calculating a safe starting dose for macromolecular agents based on the minimal anticipated biological effect level. ${ }^{27}$ Additionally, the selection of some categories of patients as subjects may bring potential risks. Concomitant diseases and medications may affect the accurate interpretation of safety data, leading to variability in the safety signals, a single or low dose may not provide sufficient therapeutic benefit to justify the inclusion of seriously ill patients in the study and the possibility of participating in subsequent trials may be ruled out. ${ }^{27}$ Finally, the seamless design of the expansion cohorts might not provide accurate information to guide future trials but rather expose a large number of patients in multiple cohorts to potentially suboptimal or toxic doses. ${ }^{25}$

In conclusion, GR1501 is expected to represent an alternative therapy option for patients with plaque psoriasis, and this study will provide safety and possibly efficacy evidence for GR1501 through this phase I/II dose escalation and expansion trial.

\section{ETHICS AND DISSEMINATION}

The study will be practiced in compliance with this study protocol. The study is in accordance with the Declaration of Helsinki, and the ethics approvals of the protocol have been obtained from the ethics committees of all participating centres (including Peking University People's Hospital, Chinese PLA General Hospital, The First Affiliated Hospital, College of Medicine, Zhejiang University and The Second Xiangya Hospital of Central South University). Before the patients are enrolled in the trial, signed informed consent will be obtained (see online supplemental appendix 5). All changes and processes in the protocol will be approved by the ethics committees.

\section{Author affiliations}

${ }^{1}$ Department of Pharmacy, Peking University People's Hospital, Beijing, China ${ }^{2}$ Department of Pharmacy Administration \& Clinical Pharmacy, School of Pharmaceutical Sciences, Peking University, Beijing, Beijing, China ${ }^{3}$ Department of Pharmacy, Xuzhou Medical University, Xuzhou, Jiangsu, China ${ }^{4}$ Department of Dermatology, Peking University People's Hospital, Beijing, China ${ }^{5}$ Genrix (Shanghai) Biopharmaceutical Co., Shanghai, China, Shanghai, China ${ }^{6}$ Department of Science and Research, Peking University People's Hospital, Beijing, China

${ }^{7}$ Department of Pharmacy, Peking University First Hospital, Beijing, China ${ }^{8}$ Center for Drug Clinical Research, Shanghai University of Traditional Chinese Medicine, Shanghai, Shanghai, China 
Acknowledgements Genrix (Shanghai) Biopharmaceutical Co., provided the drugs (GR1501). The authors would like to extend thanks to all enrolled participants and people who contributed to this study.

Contributors WD and XN: writing-original draft preparation and revising. YF, WW and WF: conception, design and plan. JW, LX, QW and TS: visualisation, investigation. QZ and HF: data curation. QW, SN, YC, JZ and LC: supervision. All authors critically reviewed the manuscript and approved the final draft for submission.

Funding This study was funded by the Chinese Society of Academic Degrees and Graduate Education 'the Project of Research of Degree and Master Education' (No:2019YX01) and Genrix (Shanghai) Biopharmaceutical Co.

Competing interests WW and WF are employees of Genrix (Shanghai) Biopharmaceutical Co.

Patient consent for publication Not required.

Provenance and peer review Not commissioned; externally peer reviewed.

Supplemental material This content has been supplied by the author(s). It has not been vetted by BMJ Publishing Group Limited (BMJ) and may not have been peer-reviewed. Any opinions or recommendations discussed are solely those of the author(s) and are not endorsed by BMJ. BMJ disclaims all liability and responsibility arising from any reliance placed on the content. Where the content includes any translated material, BMJ does not warrant the accuracy and reliability of the translations (including but not limited to local regulations, clinical guidelines, terminology, drug names and drug dosages), and is not responsible for any error and/or omissions arising from translation and adaptation or otherwise.

Open access This is an open access article distributed in accordance with the Creative Commons Attribution Non Commercial (CC BY-NC 4.0) license, which permits others to distribute, remix, adapt, build upon this work non-commercially, and license their derivative works on different terms, provided the original work is properly cited, appropriate credit is given, any changes made indicated, and the use is non-commercial. See: http://creativecommons.org/licenses/by-nc/4.0/.

ORCID iD

Wenliang Dong http://orcid.org/0000-0003-4316-1608

\section{REFERENCES}

1 Boehncke W-H, Schön MP. Psoriasis. Lancet 2015;386:983-94.

2 Parisi R, Symmons DPM, Griffiths CEM, et al. Global epidemiology of psoriasis: a systematic review of incidence and prevalence. $J$ Invest Dermatol 2013:133:377-85.

3 Burgos-Pol R, Martínez-Sesmero JM, Ventura-Cerdá JM, et al. The cost of psoriasis and psoriatic arthritis in 5 European countries: a systematic review. Actas Dermosifiliogr 2016;107:577-90.

4 Husni ME, Merola JF, Davin S. The psychosocial burden of psoriatic arthritis. Semin Arthritis Rheum 2017;47:351-60.

5 Mease PJ. Inhibition of interleukin-17, interleukin-23 and the TH17 cell pathway in the treatment of psoriatic arthritis and psoriasis. Curr Opin Rheumatol 2015;27:127-33.

6 Lynde CW, Poulin Y, Vender R, et al. Interleukin 17A: toward a new understanding of psoriasis pathogenesis. J Am Acad Dermatol 2014:71:141-50.

7 Girolomoni G, Strohal R, Puig L, et al. The role of IL-23 and the IL-23/ $T_{.} 17$ immune axis in the pathogenesis and treatment of psoriasis. $J$ Eur Acad Dermatol Venereol 2017;31:1616-26.

8 Di Cesare A, Di Meglio P, Nestle FO. The IL-23/Th17 axis in the immunopathogenesis of psoriasis. $J$ Invest Dermatol 2009;129:1339-50.
9 Hueber W, Patel DD, Dryja T, et al. Effects of AIN457, a fully human antibody to interleukin-17A, on psoriasis, rheumatoid arthritis, and uveitis. Sci Transl Med 2010;2:52ra72.

10 Baeten D, Sieper J, Braun J, et al. Secukinumab, an interleukin-17A inhibitor, in ankylosing spondylitis. N Engl J Med 2015;373:2534-48.

11 American Academy of Dermatology Work Group, Menter A, Korman $\mathrm{NJ}$, et al. Guidelines of care for the management of psoriasis and psoriatic arthritis: section 6 . guidelines of care for the treatment of psoriasis and psoriatic arthritis: case-based presentations and evidence-based conclusions. J Am Acad Dermatol 2011;65:137-74.

12 Jabbar-Lopez ZK, Yiu ZZN, Ward V, et al. Quantitative evaluation of biologic therapy options for psoriasis: a systematic review and network meta-analysis. J Invest Dermatol 2017;137:1646-54.

13 Burkett PR, Kuchroo VK. II-17 blockade in psoriasis. Cell 2016;167:1669.

14 Langley RG, Elewski BE, Lebwohl M, et al. Secukinumab in plaque psoriasis--results of two phase 3 trials. $N$ Engl J Med 2014;371:326-38.

15 Strand V, Betts KA, Mittal M, et al. Comparative effectiveness of adalimumab versus Secukinumab for the treatment of psoriatic arthritis: a Matching-Adjusted indirect comparison. Rheumatol Ther 2017;4:349-62.

16 Bai F, Li GG, Liu Q, et al. Short-term efficacy and safety of IL-17, IL12/23, and IL-23 inhibitors Brodalumab, Secukinumab, ixekizumab, ustekinumab, Guselkumab, Tildrakizumab, and Risankizumab for the treatment of moderate to severe plaque psoriasis: a systematic review and network meta-analysis of randomized controlled trials. $J$ Immunol Res 2019;2019:1-25.

17 Menter A, Strober BE, Kaplan DH, et al. Joint AAD-NPF guidelines of care for the management and treatment of psoriasis with biologics. $J$ Am Acad Dermatol 2019;80:1029-72.

18 Bissonnette R, Luger T, Thaçi D, et al. Secukinumab demonstrates high sustained efficacy and a favourable safety profile in patients with moderate-to-severe psoriasis through 5 years of treatment (SCULPTURE Extension Study). J Eur Acad Dermatol Venereol 2018;32:1507-14.

19 Reich K, Blauvelt A, Armstrong A, et al. Secukinumab, a fully human anti-interleukin-17A monoclonal antibody, exhibits minimal immunogenicity in patients with moderate-to-severe plaque psoriasis. Br J Dermatol 2017;176:752-8.

20 Blauvelt A. Safety of secukinumab in the treatment of psoriasis. Expert Opin Drug Saf 2016;15:1413-20.

21 Abstracts of the 2013 international investigative dermatology meeting. may 8-11, 2013. Edinburgh, Scotland, United Kingdom. J Invest Dermatol 2013;133:S1-311.

22 Papp KA, Langley RG, Sigurgeirsson B, et al. Efficacy and safety of secukinumab in the treatment of moderate-to-severe plaque psoriasis: a randomized, double-blind, placebo-controlled phase II dose-ranging study. Br J Dermatol 2013;168:412-21.

23 Papp KA, Reid C, Foley P, et al. Anti-IL-17 receptor antibody AMG 827 leads to rapid clinical response in subjects with moderate to severe psoriasis: results from a phase I, randomized, placebocontrolled trial. J Invest Dermatol 2012;132:2466-9.

24 Papp KA, Leonardi C, Menter A, et al. Brodalumab, an antiinterleukin-17-receptor antibody for psoriasis. N Engl J Med 2012;366:1181-9.

25 FDA. Expansion cohorts use in first-in-human clinical trials to expedite development of oncology drugs and biologics guidance for industry.[EB/OL], 2018. Available: https://www.fda.gov/media/ 115172/download [Accessed 24 Dec 2019].

26 Dorato MA, Engelhardt JA. The no-observed-adverse-effect-level in drug safety evaluations: use, issues, and definition(s). Regul Toxicol Pharmacol 2005;42:265-74.

27 The Association of the British Pharmaceutical Industry. Guidelines for phase I clinical trials 2018 edition, 2018. Available: https://www.abpi. org.uk/media/4992/guidelines-for-phase-i-clinical-trials-2018-edition20180626.pdf [Accessed 24 Dec 2019]. 